\title{
Eduardo Torroja y la International Association for Shell and Spatial Structures (IASS)
}

\section{Eduardo Torroja and the International Association for Shell and Spatial Structures (IASS)}

\author{
$\underline{\text { R. Astudillo }}^{(*)}$
}

\section{RESUMEN}

El legado de Eduardo Torroja no se limita a sus innovadores proyectos, o a sus aportaciones al cálculo y desarrollo del hormigón armado y pretensado. A lo largo de su vida, fundó, o contribuyó a fundar, diversas asociaciones, nacionales e internacionales que, como la IASS (1959), continúan vivas liderando el progreso de la construcción civil y arquitectónica.

Palabras clave: Eduardo Torroja; estructuras laminares; estructuras espaciales; hormigón; asociaciones.

\section{ABSTRACT}

Eduardo Torroja's legacy doesn't limit to his innovative designs, or to his contributions to concrete structures and their codes development. Throw his life, he founded, or contributed to found, several associations, national and international, which as IASS (1959) are working nowadays leading the progress of civil and architectural construction.

Keywords: Eduardo Torroja; shells structures; spatial structures; concrete; associations.

(*) Ing. de Caminos, Canales Puertos

Persona de contacto/Corresponding author: rastudipastor@gmail.com (R. Astudillo)

Cómo citar este artículo/Citation: Astudillo, R. (2014). Eduardo Torroja y la International Association for Shell and Spatial Structures (IASS). Informes de la Construcción, 66(536): e037, doi: http://dx.doi.org/10.3989/ic.14.113.

Licencia/License: Salvo indicación contraria, todos los contenidos de la edición electrónica de Informes de la Construcción se distribuyen bajo una licencia de uso y distribución Creative Commons Reconocimiento no Comercial 3.o. España (cc-by-nc). 


\section{LAS ASOCIACIONES INTERNACIONALES DE ESTRUCTURAS}

Otra de las facetas en las que brilló el genio de Torroja fue su destacada participación en las Asociaciones Técnicas Internacionales relacionadas con las estructuras, especialmente las estructuras laminares y los puentes, así como con los métodos de cálculo de los entonces nuevos materiales de construcción, especialmente el hormigón.

La primera asociación creada fue el IABSE (International Association for Bridges and Structural Engineering), nacida en 1922, aunque la mayoría de las demás nacieron en un muy corto periodo de tiempo posterior a la II Guerra Mundial. Así, la RILEM (Reunión Internacional de Laboratorios de Ensayo de Materiales) fue creada en 1947, el CIB (International Council for Building), la ECCS (European Convention for Constructional Steelwork), el CEB (Comité Européen du Beton) y la FIP (International Federation for Prestressing) fueron todas ellas creadas en 1952. Las dos últimas posteriormente fusionadas en la fib (Federation International du Beton).

La figura de Torroja era sobradamente respetada internacionalmente, no solamente como proyectista genial sino también por su capacidad organizativa y de liderazgo. Así, Torroja fue elegido Presidente de la RILEM en 1951, Presidente de la FIP en 1958 y considerado un destacado miembro y soporte fundamental en el CEB.

En una etapa inicial los foros de comunicación internacional en el campo de las estructuras laminares estaban incluidos dentro de ámbitos técnicos mucho más amplios; así, en los congresos de IABSE, este tipo de estructuras se trataba, como mucho, en alguna sesión técnica específica. Era patente, por tanto, la necesidad de propiciar otros foros de intercambio de ideas y conocimientos dedicados en exclusiva a estas estructuras de creciente interés.

Tras la celebración en Londres en 1952, organizado por la Cement \& Concrete Association, de un simposio sobre estructuras laminares con el nombre de «Construcción de Cubiertas Laminares de Hormigón», al que asistieron cerca de 250 miembros, aunque mayormente locales, quedó clara la necesidad de potenciar el intercambio de conocimientos y experiencias en un ámbito que fuera marcadamente internacional. El siguiente simposio, «Segundo Simposium sobre Construcción de Cubiertas Laminares de Hormigón», ya con un carácter más abierto a la participación internacional, fue organizado en Oslo, en 1957, donde se decidió la creación de un comité internacional de expertos constituido por trece miembros de distintos países para propiciar el desarrollo de las estructuras laminares. La autoridad de Torroja en este campo era tan reconocida, que fue elegido de forma unánime como presidente del citado grupo.

\section{CREACIÓN DE LA IASS}

El citado comité, reunido en Berlín, decidió también la organización en Madrid en septiembre de 1959 de un nuevo simposio, «Procesos no tradicionales de construcción de Láminas», para cuya celebración Torroja ofreció las nuevas instalaciones del Instituto Técnico de la Construcción y del Cemento, Centro que tuvo su origen en el fundado por el propio Torroja en el ámbito privado en 1934 con el nombre de Instituto Técnico de la Construcción y la Edificación, y que, tras integrarse en el Consejo Superior de Investigaciones Científicas (CSIC), se consolidó en 1949 como Instituto Técnico de la Construcción y del Cemento (ITCC), bajo la dirección de Torroja. Asimismo, en 1951 fue también nombrado Director del Laboratorio Central de Ensayo de Materiales de Construcción, perteneciente entonces a la Escuela Especial de Ingenieros de Caminos, Canales y Puertos, de la que ya era profesor desde 1939.

Así pues, bajo su dirección, existían en 1959 dos organismos: el Laboratorio Central (Ministerio de Obras Públicas) y el Instituto Técnico de la Construcción y del Cemento, (CSIC). Este último contaba desde 1953 con unas nuevas y excelentes instalaciones. Por todo ello, Torroja decidió celebrar el simposio en el ITCC, en cuyo patio trasero, y respondiendo al título del simposio, se construyeron dos modelos de cubiertas laminares prefabricadas (Figura 1). Pero lo más importante de este simposio no fueron sus resultados técnicos, sino el hecho de que durante su desarrollo se gestó definitivamente la llamada «International Association for Shell Structures» (1), cuya secretaría se estableció en el actual Laboratorio Central de Estructuras y Materiales, que desde 1957 está integrado en el CEDEX (Centro de Estudios y Experimentación de Obras Públicas) del Ministerio de Fomento.

Se decidió asimismo la creación de una revista, el Bulletin of the International Association for Shell Structures, de carácter cuatrimestral. Actualmente su nombre es el de Journal of the International Association for Shell and Spatial Structures (2), tiene carácter trimestral y, justamente en septiembre
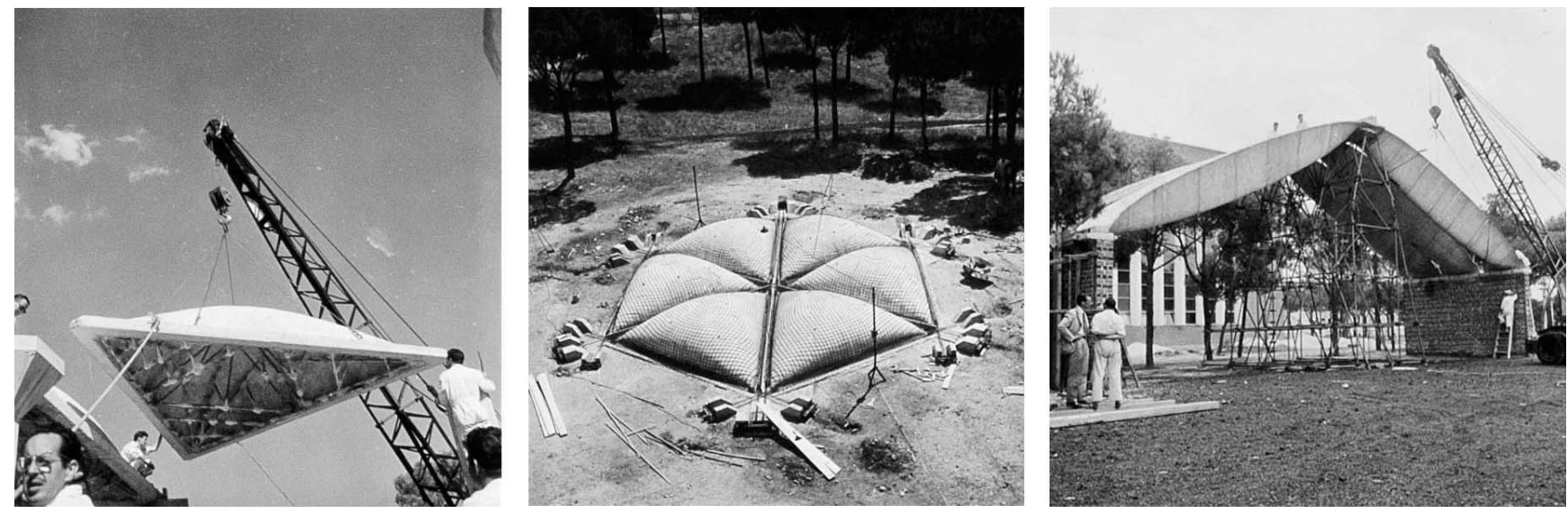

Figura 1. Estructuras experimentales construidas en el IETcc en el año 1959. Fuente: Archivo IETcc-CSIC. 
de 2014, publicó su número 181. Hay que hacer notar que a la muerte de Eduardo Torroja, siendo Presidente de la IASS el Prof. Arend M. Haas, se incorporó en la cabecera del nombre de la revista, a partir de su número 8, el subtítulo: «Prof. Dr. Ing. E. Torroja, Founder», para dejar constancia permanente de su labor y esfuerzo en la fundación de la Asociación (Figura 2).

La IASS nace pues en septiembre de 1959 con Eduardo Torroja como Presidente, los Profs. Parne (EEUU) y Olszask (Polonia) como Vicepresidentes, el Prof. Levi (Italia) como Tesorero y el Prof. Florencio del Pozo como primer Secretario de la Asociación (1).

En relación con la IASS, el IETcc, aparte de su soporte como «Maternidad» en el feliz parto de la Asociación, ha mantenido una colaboración permanente con el Laboratorio Central de Estructuras y Materiales en todos los temas en los que su concurso ha sido necesario. Los técnicos del Instituto han participado con sus trabajos en los Congresos anuales de la IASS, se han organizado visitas a sus laboratorios a petición de miembros de la IASS y ha cedido sus instalaciones para reuniones y coloquios en relación con la Asociación.

\section{LA IASS Y LAS ESTRUCTURAS ESPACIALES}

La creación de la IASS, como queda dicho, es consecuencia de la necesidad de reunir en un foro técnico internacional a un conjunto de ingenieros, arquitectos y técnicos, en general, interesados en un tipo estructural novedoso, la lámina de hormigón, que aprovechaba las posibilidades constructivas de este material y que con secciones mínimas y una nueva estética, permitía cubrir grandes espacios. Este tipo de estructuras exigían un cálculo complejo, basado muchas veces en análisis

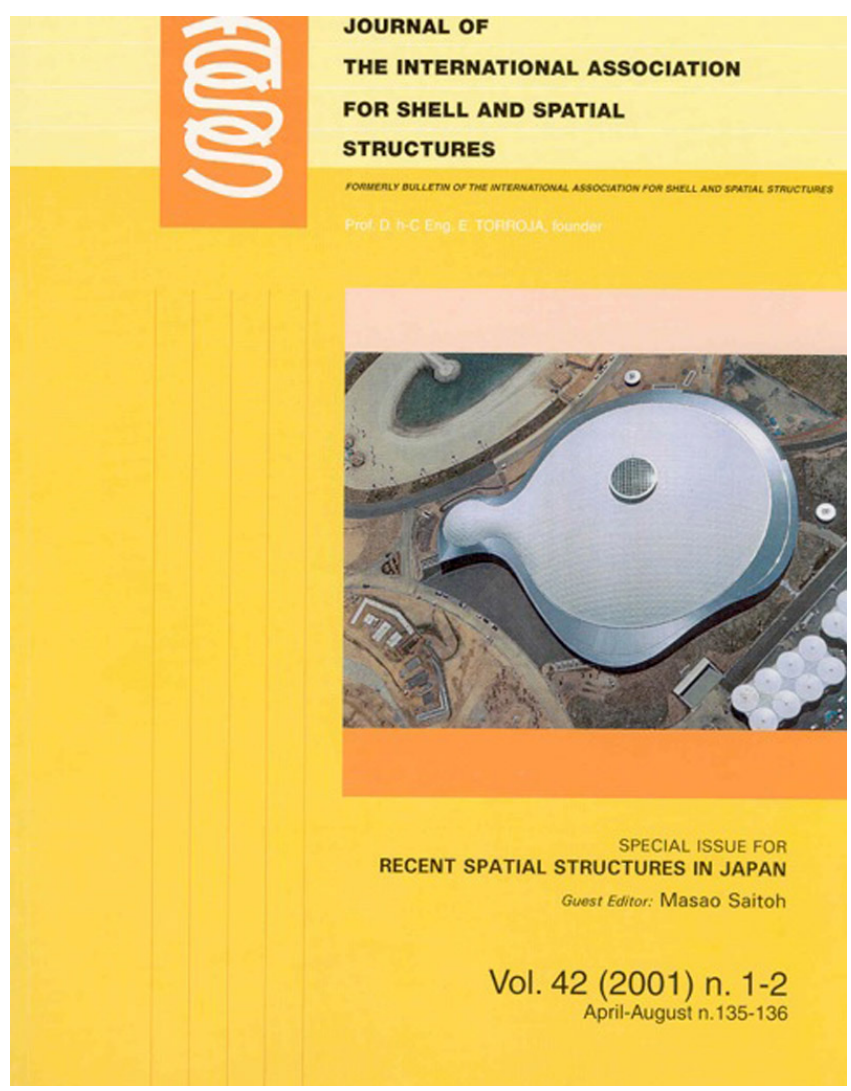

Figura 2. Portada de la revista Journal of IASS, $\mathrm{n}^{\mathrm{0}}{ }^{135^{-1}} 136$. teóricos no completamente desarrollados y contrastados, por lo que se propiciaba la necesidad del intercambio de conocimientos, siendo la IASS el foro que ofrecía tal posibilidad.

Era una época en la que los métodos numéricos y el ordenador no existían todavía como herramienta habitual del proyectista, por lo que era frecuente utilizar el modelo físico a escala reducida como método de comprobación de teorías de cálculo, o bien como medio de realización del análisis del comportamiento de estructuras concretas en su fase de proyecto. Eduardo Torroja, también pionero en la utilización del modelo reducido como método de comprobación del diseño de estructuras laminares, realizó, bien en su antiguo laboratorio de ICON o en el Laboratorio Central, numerosos modelos de láminas no solamente para diseños españoles sino también, aprovechando el foro de relación internacional que la IASS le proporcionaba, ensayos para láminas como las de la iglesia de los Santos Félix y Régula (Zurich), Bacardí (Cuba), Tachira (Caracas), entre otros.

A lo largo de los 55 años transcurridos desde su fundación, la IASS, ha ido progresando al compás de la evolución de las propias estructuras espaciales. Ya en 1971 el nombre de la Asociación International Association for Shell Structures se amplió al de International Association for Shell and Spatial Structures ya que al tiempo que decaía la utilización de las láminas de hormigón aparecían nuevos tipos estructurales que tenían acogida en el ámbito de la IASS.

\section{LAS TIPOLOGIAS ESTRUCTURALES EN LA IASS}

Aunque se han continuado utilizando posteriormente, la década de los 60 fue dominada especialmente por las láminas de hormigón (3) con señeras realizaciones de autores como A.M. Haas (Presidente de la IASS tras la muerte de Eduardo Torroja), N. Esquillian y P.L. Nervi, F. Candela (4), A. Tedesko, U. Finsterwalder y H. Isler, todos ellos Miembros de Honor de la IASS (Figura 3).
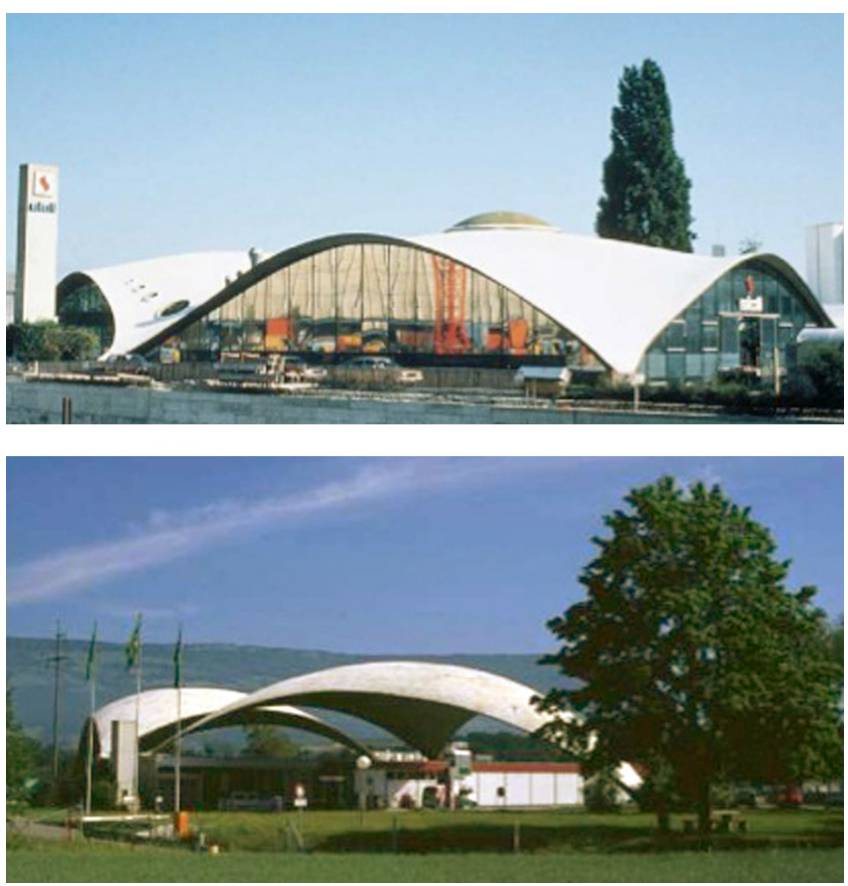

Figura 3. Arriba: Sicli Building, Geneva (1969). Abajo: Heinz Isler: Estación de servicio, Deitingen-Switzerland (1968). Fuente: Fifty Years of Progress for Shell and Spatial Structures (1). 
Incluidas también en el grupo de las láminas, aunque no exclusivamente en las de hormigón, se encuentran muchas de las estructuras utilizadas en las torres de refrigeración de centrales y los tanques y silos.

Las estructuras tensionales es otra de las tipologías habituales en el campo de interés de la IASS. Se incluyen en este tipo las estructuras de membranas textiles, las estructuras de cables y las de tensegridad, constituidas estas últimas por la combinación estable de cables en tensión y barras en compresión. Todas ellas consideradas como estructuras ligeras que se utilizan para pequeñas realizaciones que imprimen un especial carácter estético o para la cobertura de grandes espacios. Son muy frecuentes las estructuras híbridas que combinan varias tipologías de estructuras tensionales (Figura 4).

Un amplio campo de actividad en la IASS han sido las estructuras reticulares de barras de acero y nudos. Este tipo de estructuras ha evolucionado en las últimas décadas hacia la capacidad de cubrir grandes espacios, a la sistematización en la fabricación y puesta en obra de los elementos metálicos que las constituyen, así como a la libertad de formas de las cubiertas realizables con ellas. Asimismo, se progresa en su mejor comportamiento frente al sismo (Figura 5).
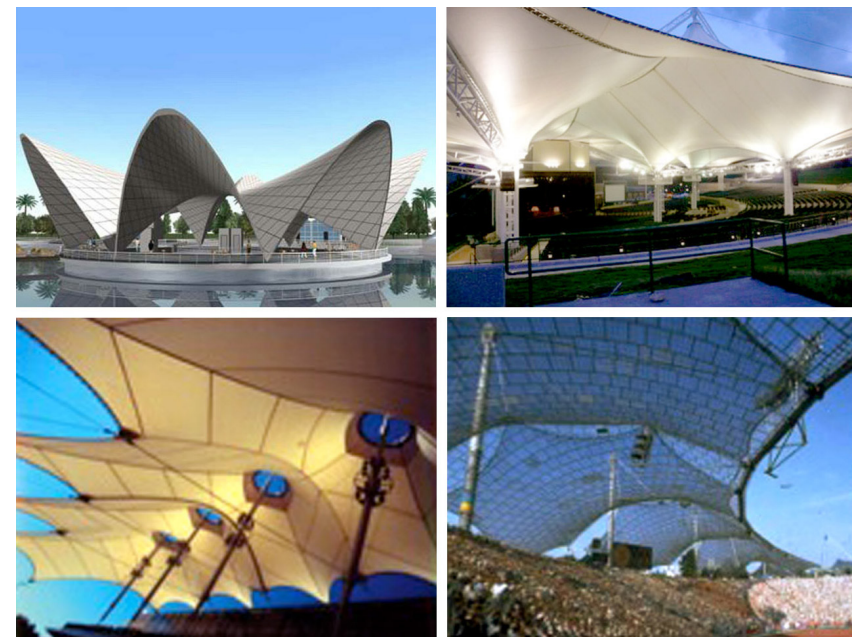

Figura 4. Arriba izq.: F. Candela: Ciudad de las Artes, Valencia. Arriba drch: Mitchell Performing, Arts Center (2009). Abajo izq.: Hampton Roads (interior). Abajo drch.: Estadio Olímpico de Munich (1972). Fuente: Fifty Years of Progress for Shell and Spatial Structures (1).

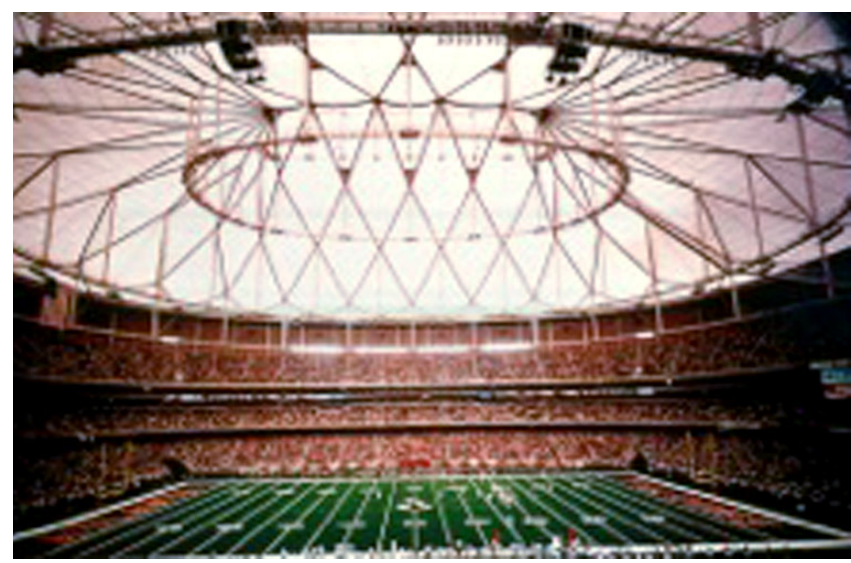

Figura 5. Matthys Levy: Georgia Dome, Weidlinger Associates (1992). Fuente: Fifty Years of Progress for Shell and Spatial Structures (1).
Aunque no frecuentes en España si lo son en muchas áreas geográficas las estructuras de madera. En la IASS quedan adecuadamente tratadas en uno de los Comités Técnicos, el WG 12.

Otros tipos estructurales mas específicos pero que tienen cabida en la IASS son las estructuras desplegables (permiten su plegado y desplegado) en las que el recientemente fallecido Félix Escrig (5), miembro del Comité Ejecutivo de la IASS, era una autoridad internacional (Figura 6).

Las Estructuras de vidrio nacen de la combinación de las celosías metálicas con el cristal y se vienen utilizando con profusión en los últimos años, dando lugar a realizaciones de gran belleza estética y funcionalidad (Figura 7).

Las torres y mástiles también constituye un prolífico grupo de trabajo en la IASS con una importante actividad que se traduce en la organización frecuente de coloquios específicos sobre este tipo de estructuras.
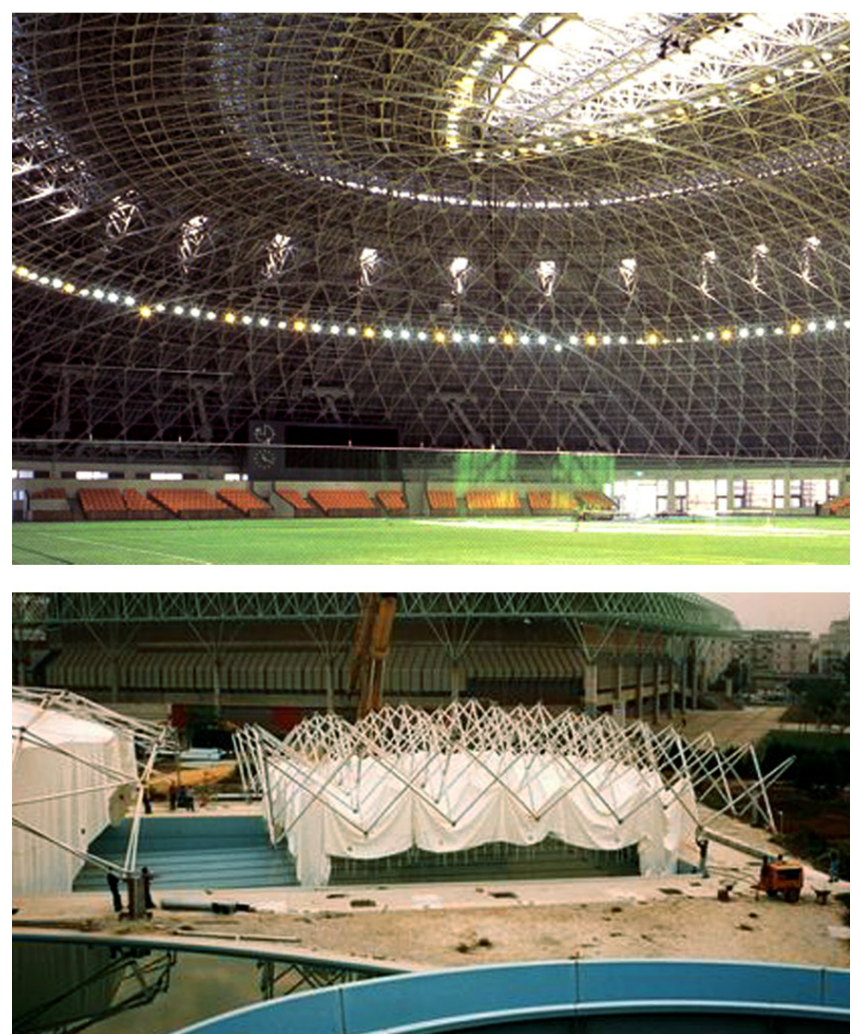

Figura 6. Arriba: Celosía espacial. Abajo: Félix Escrig: Cubierta desplegable para piscina, Sevilla. Fuente: Fifty Years of Progress for Shell and Spatial Structures (1)

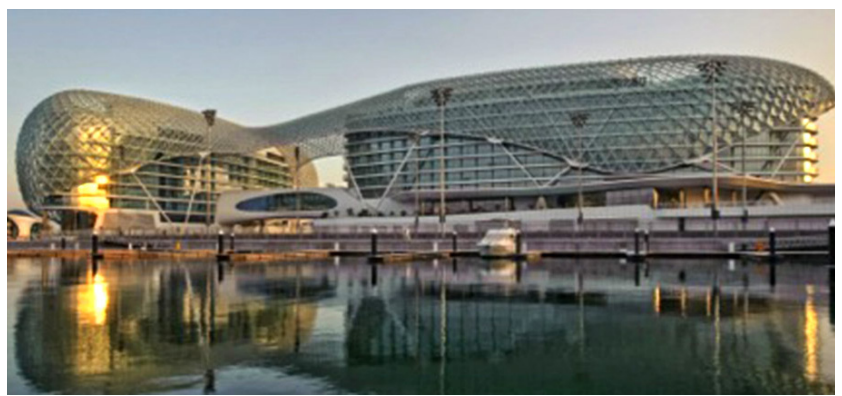

Figura 7. Estructura de vidrio con forma libre. Fuente: Fifty Years of Progress for Shell and Spatial Structures (1). 


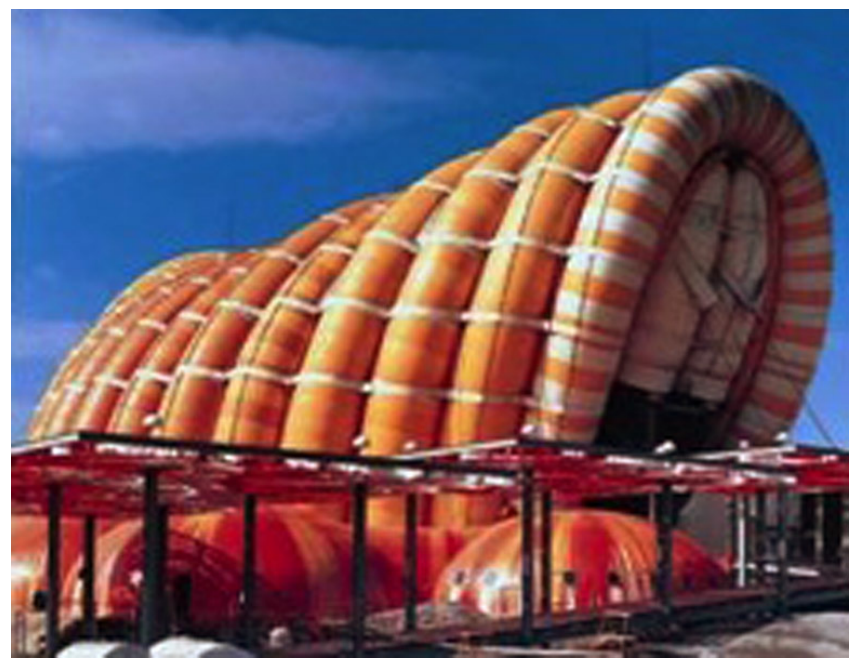

Figura 8. Fuji Group Pavilion. Fuente: Fifty Years of Progress for Shell and Spatial Structures (1).

Las estructuras temporales también son consideradas en el ámbito de trabajo de la IASS, incluyéndose dentro de ellas las estructuras neumáticas (Figura 8).

\section{DETALLES DE LA ASOCIACIÓN}

En la actualidad la IASS esta constituida como una asociación sin ánimo de lucro e inscrita en el Registro Español de Asociaciones. El idioma de trabajo es el inglés.

\section{Miembros}

Cuenta con cerca de 800 miembros de más de 50 países, siendo Estados Unidos, Japón, China, Alemania y España, por este orden, los que tienen un mayor número de miembros. Existen varias categorías de miembros, siendo las principales las de miembros Individuales, Colectivos, Subscriptores, Estudiantes y Chapters. Estos últimos son grupos de miembros en países con bajo nivel de renta, que gracias a una cuota especialmente reducida, pueden pertenecer a la Asociación y recibir de forma electrónica la revista de la IASS.

\section{Organización}

La organización administrativa está constituida por un Presidente, cuatro Vicepresidentes, un Tesorero y un Secretario. Uno de los Vicepresidentes y el Secretario pertenecen al Laboratorio Central del CEDEX, que acoge la Secretaría. Actualmente, René Motro (Francia) es el Presidente, J.M. Gálligo (España), K. Kawaguchi (Japón), C. Lázaro (España) y S. Pellegrino (EEUU) son Vicepresidentes, R. Shaeffer (EEUU) es Tesorero y J. Mora (España) es Secretario.

El órgano máximo de decisión es el Comité Ejecutivo con 24 miembros. Cada año se renuevan seis de los miembros del Comité mediante elección directa por todos los miembros activos de la Asociación. El Comité se reúne una vez al año coincidiendo con el Congreso Anual de la IASS.

\section{Grupos de trabajo}

Los grupos de trabajo actualmente activos son los que figuran en la Tabla 1.
Tabla 1. Grupos de trabajo activos de la IASS.

\begin{tabular}{|l|l|}
\hline \multicolumn{2}{|c|}{ GRUPOS DE TRABAJO ACTIVOS } \\
\hline WG 3 & Cooling and Solar Updraft Towers \\
\hline WG 4 & Masts and Towers \\
\hline WG 5 & Concrete Shell Roofs \\
\hline WG 6 & Tension and Membrane Structures \\
\hline WG 8 & Metal Spatial Structures \\
\hline WG 12 & Timber Spatial Structures \\
\hline WG 13 & Computational Methods \\
\hline WG 15 & Structural Morphology \\
\hline WG 17 & Historical Spatial Structures \\
\hline WG 18 & Environmentally Compatible Structures \\
\hline WG 19 & Temporary Spatial Structures \\
\hline
\end{tabular}

\section{Publicaciones}

Anualmente se publican cuatro números de la revista de la IASS: Journal of the International Association for Shell and Spatial Structures, que son editados en el Laboratorio Central de Estructuras y Materiales y en los que se recogen y diseminan los estudios, experimentos, proyectos y realizaciones en relación con todos los aspectos de las estructuras que constituyen el campo de la Asociación.

La revista está a su vez accesible para todos los miembros en la página web de la Asociación (6). Asimismo, se publican recomendaciones técnicas elaboradas por los grupos de trabajo que se distribuyen de forma gratuita a todos los miembros.

\section{Premios y reconocimientos de la Asociación}

- Medalla Torroja: Concedida a técnicos de relevancia internacional que hayan destacado de forma especialmente notoria en el campo de las estructuras espaciales (Figura 9).

- Miembro de Honor: Concedido a personas con relevantes aportaciones en la actividad de la IASS o en el desarrollo de los fines de la Asociación.

- Premios Tsuboi: Otorgados cada año, uno, al mejor artículo publicado en el «Journal» en el año anterior y otro, al mejor trabajo presentado en el Congreso anual del año anterior.

- Premios Hangai: Otorgados cada año a los mejores trabajos de autores jóvenes que participan en el Congreso Anual y que se registran para optar al premio.

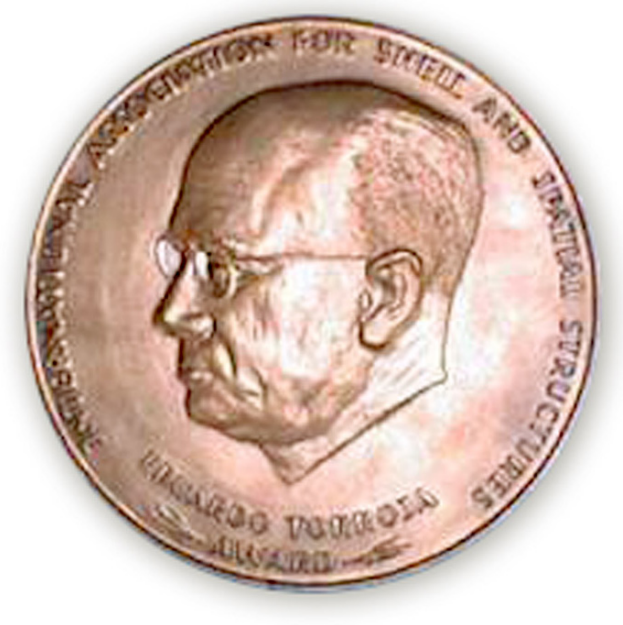

Figura 9. Medalla Torroja. 


\section{Liaison Committee}

La IASS pertenece a un Comité Internacional de Asociaciones Técnicas en el campo de la construcción constituido por las siguientes: ECCS, CIB, fib, IABSE y RILEM. El Comité se reúne una vez al año para coordinar las actividades de las asociaciones evitando duplicidades, así como para tratar temas de interés común.
En definitiva, la IASS, desde su creación por Eduardo Torroja, es uno de los escasos foros técnicos de ámbito mundial cuya cabeza organizativa reside permanentemente en España, lo que constituye una vía privilegiada para recibir, intercambiar y, especialmente, proyectar nuestros logros técnicos en el campo de las estructuras hacia el resto de la comunidad científica internacional.

\section{REFERENCIAS}

(1) Mungan, I., Abel, J.F. (2011). Fifty Years of Progress for Shell and Spatial Structures. Reino Unido: Multi-Science Publishing Co. Ltd. Essex.

(2) Announcement by the Organizing Committee (Eds.). (2014). IASS Symposium 2015. Amsterdam. Aug 17-20, 2015. Journal of the International Association for Shell and Spatial Structures, 55(181): 142.

(3) García, R. (2013). Dos décadas de estructuras plegadas de hormigón. Inicio y ocaso de un movimiento. Informes de la Construcción, 65(529): 27-39, doi: http://dx.doi.org/10.3989/ic.11.083.

(4) Cassinello, P., Schlaich, M., Torroja, J. A. (2010). Félix Candela. En memoria (1910-1997). Del cascarón de hormigón a las estructuras ligeras del s. XXI. Informes de la Construcción, 62(519): 5-26 doi: http://dx.doi.org/10.3989/ic.10.040.

(5) Escrig, F. (1984). Estructuras espaciales de barras desplegables. Informes de la Construcción, 36(365): 35-46, doi: http:// dx.doi.org/10.3989/ic.1984.v36.i365.1895.

(6) IASS. http://www.iass-structures.org/index.cfm/page/Hist/JB.htm. Madrid: The International Association for Shell and Spatial Structures. 